\section{TIN: Radiotherapie bleibt Standard}

\section{Bei fast jedem zehnten Patienten mit einem Keimzelltumor eines Hodens finden sich kontralateral testikuläre intraepitheliale Neoplasien. In einer aktuellen Studie wurde untersucht, wie sich diese am effektivsten behandeln lassen.}

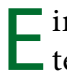
ne deutsche Studiengruppe hat untersucht, welche Therapien für testikuläre intraepitheliale Neoplasien (TIN), Vorläufer weiterer Keimzelltumoren, am besten sind. Die Autoren werteten retrospektiv die Daten von 228 Patienten mit einseitigen Keimzelltumoren aus, bei denen die Histologie im kontralateralen Hoden den Befund einer TIN bestätigt hatte. An 64 urologischen Zentren in Deutschland und Österreich wurden diese TIN entsprechend den geltenden Standards behandelt.

122 Patienten erhielten eine Radiotherapie dieses Hodens mit einer Strahlendosis zwischen 18 Gy und 20 Gy. In 30 Fällen wurde eine Chemotherapie mit maximal zwei Zyklen (Cisplatin-Etoposid-Bleomycin) durchgeführt, in 51 Fällen mit mindestens drei Zyklen dieses
Schemas. Eine Monotherapie mit Carboplatin (bis zwei Zyklen, AUC-Wert: 7) erhielten 15 Patienten. Zehn Studienteilnehmer wurden nur engmaschig kontrolliert. Primärer Studienendpunkt war ein malignes Ereignis, definiert als Diagnose einer TIN bei einer Kontrollbiopsie oder eines Keimzelltumors in der weiteren Beobachtungszeit.

In der Gruppe mit Radiotherapie wurde bei 2,5\% der Studienteilnehmer ein malignes Ereignis festgestellt; das mediane ereignisfreie Überleben lag hier bei 11,1 Jahren. Nach zwei Zyklen der kombinierten Chemotherapie betrug die Ereignisrate $50 \%$, das ereignisfreie Überleben 3,0 Jahre. Die entsprechenden Werte für drei oder mehr Zyklen lagen bei $24 \%$ beziehungsweise 9,8 Jahren. Eine Carboplatin-Monothe- rapie war mit einer Ereignisrate von $66 \%$ und einer ereignisfreien Überlebenszeit von 0,9 Jahren verbunden. Unter reiner Kontrolle wurden in $50 \%$ TIN oder Keimzelltumoren bei einem ereignisfreien Überleben von 7,1 Jahren beobachtet.

Hypogonadismus trat unter den verschiedenen Behandlungen mit folgenden Raten auf: $31 \%$ (Radiotherapie), $13 \%$ (bis zwei Zyklen Kombinationsschema), $18 \%$ (drei oder mehr Zyklen), $40 \%$ Carboplatin, $40 \%$ (Kontrollbeobachtung).

Fazit: Die Radiotherapie führte bei TIN mit Abstand zu den besten Überlebensdaten. Auch wenn bei einem Drittel der Patienten Hypogonadismus beobachtet wurde, empfehlen die Autoren diese Behandlung als Standard. Dr. Andreas Fischer

Dieckmann K-P et al. Treatment of testicular intraepithelial neoplasia (intratubular germ cell neoplasia unspecified) with local radiotherapy or with platinum-based chemotherapy: a survey of the German Testicular Cancer Study Group.

Ann Oncol 2013; 24: 1332-7

\section{Blasenkatheter-Entfernung: Antibiotika-Prophylaxe für Risiko-Patienten?}

\section{Patienten, die aufgrund einer Operation kurzzeitig mit einem \\ Blasenkatheter versorgt werden, entwickeln seltener einen symptomatischen Harnwegsinfekt, wenn sie bei dessen Entfernung eine Antibiotikaprophylaxe erhalten. Doch diese Maßnahme kann aus vielerlei Gründen keine gängige Praxis sein.}

B ei chirurgischen Patienten ist eine vorübergehende Katheterisierung Alltag. Trotz der Empfehlung, den Urinkatheter innerhalb von 24 bis 48 Stunden nach der Operation zu entfernen, birgt dieser für den Patienten ein erhöhtes Risiko für eine Harnwegsinfektion. Nicht nur während der Katheter liegt, auch bei dessen Entfernung können Bakterien in die Harnwege gelangen und eine Infektion verursachen. Ob hier eine prophylaktische Gabe von Antibiotika sinnvoll ist, wird bislang kontrovers diskutiert. Mit einem systematischen Review hat ein US-amerikanisches Autorenteam nun versucht, die Spreu vom
Weizen zu trennen. Übrig blieben sieben Studien mit 1.520 Patienten.

Die Auswertung zeigte einen Vorteil für die Probanden, die Antibiotika erhielten. Ihr absolutes Risiko für eine Harnwegsinfektion war um 5,8\% niedriger als das in der Kontrollgruppe ohne eine solche Prophylaxe (Infektionsraten $5,5 \%$ vs. $10,5 \%)$. Um eine symptomatische Harnwegsinfektion zu verhindern, mussten 17 Patienten antibiotisch behandelt werden („number needed to treat", NNT).

Fazit: Mit einer routinemäßigen Antibiotikaprophylaxe bei der Katheterent- fernung ließe sich zwar das Risiko für Harnwegsinfektionen senken, diese Maßnahme brächte allerdings auch einen Berg neuer Probleme mit sich, wie Nebenwirkungen, die Verbreitung von Resistenzen (insbesondere gegen Trimethoprim/Sulfamethoxazol und Ciprofloxacin) sowie enorme zusätzliche Kosten. Diese könnten nach Ansicht der Autoren durch eine strenge Patientenselektion gemildert werden, sodass nur diejenigen Patienten die Maßnahme erhalten, die am meisten davon profitieren. In künftigen Studien sollte deshalb untersucht werden, welche Personen am meisten gefährdet sind, nach der Entfernung eines Katheters eine Harnwegsinfektion zu entwickeln. Auf diese Personen könnte sich die Antibiotikaprophylaxe dann konzentrieren. Dr. Christine Starostzik

Marschall J et al. Antibiotic prophylaxis for urinary tract infections after removal of urinary catheter: meta-analysis. BMJ 2013; 346: f3147 